\title{
A comparison of indirect calorimeters' measures of resting metabolic rate
}

\author{
S. Kennedy, A. Fraser, L. Ryan and M. E. Clegg \\ Functional Food Centre, Department of Sport and Health Sciences, Oxford Brookes University, Oxford, UK
}

Resting metabolic rate (RMR) represents the energy required to sustain vital bodily functions while resting. RMR comprises the largest component of total energy expenditure $(60-75 \%)$ in sedentary people ${ }^{(1)}$ and is calculated in clinical and research settings using indirect calorimetry. The Deltatrac ${ }^{(i x}$ II Metabolic Monitor (Datex-Ohmeda Inc., Finland) has been well-established as the reliable reference system in indirect calorimetry for over fifteen years ${ }^{(2)}$, however, it is no longer being manufactured thus there is a need to validate new indirect calorimeters that closely mimic the Deltatrac's reliability. The GEM (Gas Exchange Measurement, GEMNutrition Ltd, UK) and the ECAL (Energy Testing Solutions Ltd, Australia) are alternative measuring systems. The aim of this study was to compare the accuracy and repeatability of measured RMR values between three indirect calorimeters.

Twenty healthy participants (16 female, 4 male; mean age: 27yrs (SD 8); mean height: $1.7 \mathrm{~m}$ (SD 0.1); mean weight: 66.2 kg (SD 11.9)) were tested on two occasions following an overnight fast. On the first test day, participants' height, weight and \% body fat were measured. Following 30 minutes rest, RMR was measured for 30 minutes on the Deltatrac, the GEM and the ECAL in a randomised order on two non-consecutive days. $\mathrm{VO}_{2}$ and $\mathrm{VCO}_{2}$ data generated from all three machines was collected and used to calculate RMR using the Lusk equation ${ }^{(3)}$. Data were compared using Pearson correlation and Bland-Altman analysis.

There were significant correlations $(p<0.01)$ within machines and between test days (Table 1), however, the GEM and the ECAL reported higher RMR values in comparison to the Deltatrac (Table 2). Between-test repeatability saw the greatest difference and variation within the ECAL (Table 2). The ECAL had the closest mean RMR values (1544 kcal/d, SD 458) to the Deltatrac (1362 kcal/d, SD 220$)$ but showed greater variation than the GEM (1691 kcal/d, SD 245).

Table 1. Deltatrac, ECAL \& GEM RMR correlations within tests and between tests (repeatability)

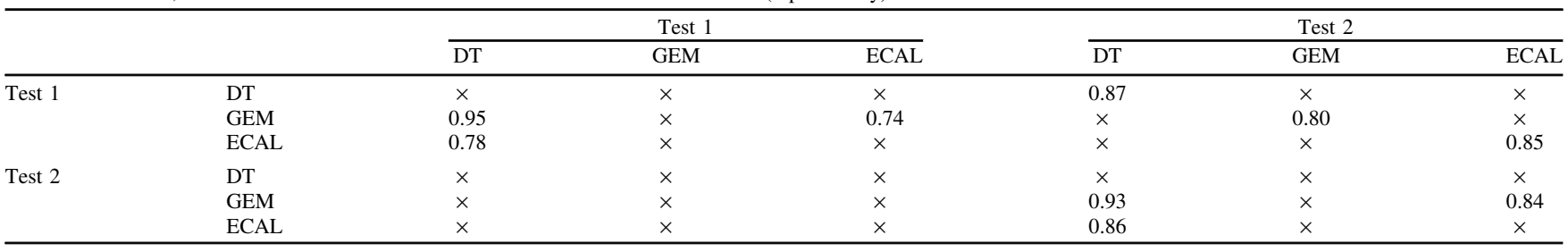

Table 2. Deltatrac, ECAL \& GEM mean RMR differences (kcal/day) within tests \& between tests (repeatability)

\begin{tabular}{|c|c|c|c|c|c|c|c|c|c|c|c|c|c|}
\hline & & \multicolumn{6}{|c|}{ Test 1} & \multicolumn{6}{|c|}{ Test 2} \\
\hline & & DT & SD & GEM & SD & ECAL & SD & DT & SD & GEM & SD & ECAL & $\mathrm{SD}$ \\
\hline \multirow[t]{3}{*}{ Test 1} & DT & $\times$ & $\times$ & $\times$ & $\times$ & $\times$ & $\times$ & 31.1 & 116.9 & $\times$ & $\times$ & $\times$ & $\times$ \\
\hline & GEM & 314.1 & 64.3 & $\times$ & $\times$ & 204.2 & 369.9 & $\times$ & $\times$ & -2.4 & 167.5 & $\times$ & $x$ \\
\hline & ECAL & 109.9 & 360.6 & $x$ & $\times$ & $\times$ & $\times$ & $x$ & $\times$ & $\times$ & $\times$ & 113.4 & 258.3 \\
\hline \multirow[t]{3}{*}{ Test 2} & DT & $\times$ & $\times$ & $\times$ & $x$ & $x$ & $\times$ & $\times$ & $\times$ & $\times$ & $\times$ & $\times$ & $\times$ \\
\hline & GEM & $\times$ & $\times$ & $\times$ & $\times$ & $\times$ & $\times$ & 342.8 & 107.3 & $\times$ & $\times$ & 88.3 & 239.2 \\
\hline & ECAL & $\times$ & $x$ & $\times$ & $x$ & $x$ & $x$ & 254.5 & 247.4 & $\times$ & $\times$ & $\times$ & $\times$ \\
\hline
\end{tabular}

The current data indicates that differences in daily RMR existed between all three machines. These differences were however in keeping with that seen previously in the literature ${ }^{(4)}$. All three machines showed good day-to-day repeatability.

1. Harris JA \& Benedict FG (1919) In A biometric study of basal metabolism in man. Washington DC: Carnegie Institute of Washington.

2. Alam DS, Hulshof PJ, Roordink D et al. (2005) Eur J Clin Nutr 59, 651-7.

3. Lusk G (1928) In The Science of Nutrition. W.B Saunders Co, London.

4. Littlewood RA, White MS, Bell KL et al. (2002) Clin Nutr 21(6), 491-497. 